\title{
Mitochondrial medicine
}

\author{
Saskia Koene • Jan Smeitink
}

Published online: 2 March 2011

(C) The Author(s) 2011. This article is published with open access at Springerlink.com

\begin{abstract}
Almost 50 years after the first description of a patient with a mitochondrial disease (Luft et al. 1962), there now is an extraordinarily rapid development in mitochondrial medicine. In the first 30 years of mitochondrial disease research, the focus was centered on unravelling the aetiology of the high variety of manifestations of mitochondrial dysfunction. Currently, one of the most important focuses is on providing a cure for these devastating diseases. In the first chapter of this issue, Salvatore DiMauro will guide you through the history of mitochondrial medicine, chronologically discussing important discoveries and dedicated mitochondriacs diseases (DOI: 10.1007/s10545-010-9082-x). Besides the ongoing development of new techniques to study mitochondrial (patho)physiology, genetics and organelle dynamics, it is the individual patients that taught us most about the physiology of the mitochondria.

Identifying mitochondrial disease patients and subsequently establishing the diagnosis is one of the clinical challenges in mitochondrial medicine, because of the clinical heterogeneity and the ever-expanding phenotype. Before the diagnosis can be confirmed by biochemical analysis of fresh tissue acquired by a muscle biopsy, the indication of the muscle biopsy must be set on clinical and biochemical grounds, supported by the use of biomarkers. Anu Suomalainen describes the various parameters which can be used to support the clinical diagnosis of mitochondrial disease (DOI: 10.1007/s10545-010-9222-3). Unfortunately,
\end{abstract}

\footnotetext{
S. Koene $\cdot$ J. Smeitink $(\bowtie)$

Radboud University Nijmegen Medical Centre,

Nijmegen, The Netherlands

e-mail: J.Smeitink@cukz.umcn.nl

S. Koene

e-mail: s.koene@cukz.umcn.nl
}

most biomarkers used in every day practice, such as lactate and alanine, are not $100 \%$ sensitive and specific. Various new approaches are described, such as the measurement of intracellular glutathione levels, proteomics and lipidomics.

After the clinical suspicion of mitochondrial disease, it is important to confirm the diagnosis by biochemical examination of fresh muscle tissue. The techniques used to analyze mitochondrial function are more and more automated and therefore become quicker. To confirm a primary mitochondrial dysfunction, a mutation in one of the structural or facilitating proteins should be confirmed. Previously, detecting and proving the pathogenicity of a DNA mutation, either in nuclear or in mitochondrial DNA, required a huge amount of labor. Thanks to many enduring researchers, numerous mutations in mitochondrial DNA (mtDNA) and nuclear DNA have been found. Since the commercial availability of the MitoChip, the sequencing velocity of mtDNA has increased immensely and many new mutations and polymorphisms are found constantly. In nuclear DNA, the new high-throughput DNA sequencing methods along with bioinformatical analysis will certainly speed up the identification of new genes and mutations (DOI: 10.1007/s10545-010-9081-y).

With the identification of mutations in nuclear genes, it has become possible to create an animal model with a mutation similar to the gene defect seen in patients. Two mouse models have been created with an isolated complex I deficiency, which is the most common biochemical defect in mitochondrial disease (DOI: 10.1007/s10545-009-9005-x). Both models show a striking similarity with the clinical and biochemical phenotype seen in human complex I patients.

Many advanced technologies have been used to design the perfect drug to combat mitochondrial disease. Most of the experiments are still at the cellular level. Several strategies have been practiced/practised to develop mito- 
chondrial treatment options (Koene \& Smeitink 2009), namely altering the balance between mutated and healthy DNA, metabolic manipulation and transcription regulation, gene therapy and the prevention of transmission of mtDNA mutations.

Most of the above mentioned approaches are only applicable in mtDNA mutations, as is the case for the attempts to alter the balance between mutated and healthy mtDNA (heteroplasmy) (DOI: 10.1007/s10545-010-9122-6). Since cellular dysfunction occurs only when the threshold for heteroplasmy is exceeded, stimulating replication of the healthy mtDNA or reducing the mutated mtDNA will potentially increase cellular function. Smith and Lightowlers describe several ways to manipulate heteroplasmy by, for instance, exercise training or genetic manipulation.

Another rational way to combat mitochondrial dysfunction is to correct the metabolic alterations seen at the cellular level (DOI: 10.1007/s10545-010-9162-y). Since these alterations are seen in both mitochondrial and nuclear mutations as well as secondary mitochondrial dysfunction, this approach is theoretically applicable in all mitochondrial diseases. In the past decade, the consequences of oxidative phosphorylation dysfunction, such as a increased production of reactive oxygen species, have been mapped in detail and automated quantitative live-assays have been developed to visualize these parameters (Koopman et al. 2009). Many research groups around the world have established ways to manipulate mitochondrial pathology in cellular models. Now is the time to test these compounds in vivo.

Gene therapy to minimize the negative impact of mtDNA mutations on cell viability has also shown promising results at the cellular level (DOI: 10.1007/s10545010-9131-5). The most promising ways to manipulate the genetic environment of the mitochondria are afforded by using import of wild-type copies of DNA or RNA into mitochondria, manipulating the mitochondrial genetic content or rescuing a defect by expressing an engineered gene product from the nucleus (allotopic or xenotopic expression). However, utilization of these techniques in in vivo studies is frustrated by a lack of reliable animal models and difficulties in the delivery and expression of the therapeutic gene.
Despite the lack of a cure, it is important to recognize mitochondrial disorders as they are a frequent cause of inborn errors of metabolism. Therefore, it is important to create mitochondrial-awareness among doctors, to detect and diagnose the mitochondriopathy. Despite the apparent lack of therapy, the confirmation of a mitochondrial disease is important to prevent secondary mitochondrial dysfunction as seen in malnutrition or deconditioning (Morava et al. 2006).

The confirmation of a diagnosis can also be of great importance in family planning, since there are experimental ways to prevent transmission of mtDNA mutations and to perform prenatal diagnostics in patients with nuclear mutations. As long as we cannot cure mitochondrial diseases, this approach is of great value for parents having a child with mitochondrial disease.

This issue will give an overview of some hopeful breakthroughs and the many challenges remaining in pursuit of the first effective treatment for mitochondrial disease. There seems to be light at the end of the tunnel, although currently it is difficult to predict when such treatment will become available.

Open Access This article is distributed under the terms of the Creative Commons Attribution Noncommercial License which permits any noncommercial use, distribution, and reproduction in any medium, provided the original author(s) and source are credited.

\section{References}

Luft R, Ikkos D, Palmieri G, Ernster L, Afzelius B (1962) A case of severe hypermetabolism of nonthyroid origin with a defect in the maintenance of mitochondrial respiratory control: a correlated clinical, biochemical, and morphological study. J Clin Invest 41:1776-1804

Koene S, Smeitink J (2009) Mitochondrial medicine: entering the era of treatment. J Intern Med 265:193-209

Koopman WJ, Nijtmans LG, Dieteren CE, Roestenberg P, Valsecchi F, Smeitink JA, Willems PH (2009) Mammalian mitochondrial complex I: Biogenesis, regulation and reactive oxygen species generation. Antioxid Redox Signal

Morava E, Rodenburg R, van Essen HZ, De Vries M, Smeitink J (2006) Dietary intervention and oxidative phosphorylation capacity. J Inherit Metab Dis 29:589 